Preprint typeset in JHEP style - HYPER VERSION

DFTT 48/2009

\title{
On the Sunyaev-Zel'dovich effect from dark matter annihilation or decay in galaxy clusters
}

\author{
Julien Lavalle \\ Dipartimento di Fisica Teorica, Università di Torino ES INFN, via Giuria 1, 10125 \\ Torino - Italia \\ Email: lavalle@to.infn.it \\ Céline Bœhm \\ LAPTH, UMR 5108, 9 chemin de Bellevue - BP 110, 74941 Annecy-Le-Vieux - France \\ Email: boehm@lapp.in2p3.fr \\ Julien Barthès \\ LAPTH, UMR 5108, 9 chemin de Bellevue - BP 110, 74941 Annecy-Le-Vieux - France \\ Email: barthes@lapp.in2p3.fr
}

\begin{abstract}
We revisit the prospects for detecting the Sunyaev Zel'dovich (SZ) effect induced by dark matter (DM) annihilation or decay. We show that with standard (or even extreme) assumptions for DM properties, the optical depth associated with relativistic electrons injected from DM annihilation or decay is much smaller than that associated with thermal electrons, when averaged over the angular resolution of current and future experiments. For example, we find: $\tau_{\mathrm{DM}} \sim 10^{-9}-10^{-5}$ (depending on the assumptions) for $m_{\chi}=1 \mathrm{GeV}$ and a density profile $\rho \propto r^{-1}$ for a template cluster located at $50 \mathrm{Mpc}$ and observed within an angular resolution of $10^{\prime \prime}$, compared to $\tau_{\text {th }} \sim 10^{-3}-10^{-2}$. This, together with a full spectral analysis, enables us to demonstrate that, for a template cluster with generic properties, the SZ effect due to DM annihilation or decay is far below the sensitivity of the Planck satellite. This is at variance with previous claims regarding heavier annihilating DM particles. Should DM be made of lighter particles, the current constraints from $511 \mathrm{keV}$ observations on the annihilation cross section or decay rate still prevent a detectable SZ effect. Finally, we show that spatial diffusion sets a core of a few $\mathrm{kpc}$ in the electron distribution, even for very cuspy DM profiles, such that improving the angular resolution of the instrument, e.g. with ALMA, does not necessarily improve the detection potential. We provide useful analytical formulæ parameterized in terms of the DM mass, decay rate or annihilation cross section and DM halo features, that allow quick estimates of the SZ effect induced by any given candidate and any DM halo profile.
\end{abstract}

KEYWORDS: Sunyaev-Zel'dovich effect; dark matter theory; galaxy clusters. 


\section{Contents}

1. Introduction

2. Principle 2

3. Injected electron density from DM annihilation or decay and optical depths

3.1 Line-of-sight averaged optical depth and electron density

3.2 Analytical approximations 8

3.2.1 The spherical approximation 8

3.2.2 The line-of-sight approximation 9

3.3 Thermal to DM optical depth ratio $\eta_{\chi}$

4. Spectral distortion analysis 11

5. Impact of spatial diffusion: analytical insights 19

6. Conclusion 22

\section{Introduction}

The enigma of the origin of dark matter (DM) is a longstanding issue. Many experimental strategies in particle physics, astrophysics and cosmology have been developed to confirm/infirm the existing scenarios. One appealing possibility, strongly motivated by independent issues in particle physics beyond the standard model (BSM), is that DM is made of weakly interacting particles (WIMPs). The most popular models, like the supersymmetric (SUSY) neutralino (see e.g. [1, 2] for reviews), have the interesting property of self-annihilation to ordinary matter, which provides windows for the detection of the annihilation products. There is actually an impressive wealth of WIMP models, from the $\mathrm{GeV}-\mathrm{TeV}$ mass-scale as in the SUSY paradigm down to the sub-GeV mass-scale, the latter class allowing, in particular, to solve the so-called cusp and subhalo problems (e.g. [3]). Since the DM annihilation or decay rate increases with the DM number density, the best targets are expected to be the centers of (sub)galaxies or galaxy clusters. This quest for annihilation or decay signals makes use of different messengers (e.g. $\gamma$-rays, antimatter cosmic rays and neutrinos): it is referred to as indirect detection of DM (for a pedagogical review, see [4]). There are of course other ways to learn about the microscopic properties of DM, for instance by detecting direct interactions of DM in ground based experiments «, or by producing DM directly in particle colliders. All these direct and indirect detection methods have been studied at length in the literature. 
Yet, a few years ago, it was pointed out in a series of papers [5, 6, 7] a new complementary method to look for DM, namely the Sunyaev-Zel'dovich (SZ) effect [8, 9]. Indeed, DM annihilation or decay in galaxy clusters may inject relativistic electrons and positrons ${ }^{1}$ that should experience inverse Compton scattering with the CMB photons and, therefore, if numerous enough, generate a deviation to the blackbody spectrum. The question that we address in this paper is whether or not this deviation is observable in light of the contradictory results found by [5, 6, [] on the one hand, and by [10] on the other hand. In particular, we investigate whether the Planck satellite [1], which was recently launched, and the ALMA facilities 12, 13 will have the sensitivity to detect the SZ signal induced by DM annihilation or decay and could constrain the DM properties.

Our approach aims at providing more analytical insights than 10 on the crucial impact of the experimental angular resolution on the DM-induced SZ predictions, on the spectral analysis itself and on spatial diffusion effects. We also consider the SZ effect due to the thermal electrons lying in clusters and observed in X-ray measurements, which has been studied for a long time (see [14] for a review) and which constitutes an important background to such an exotic signature. To proceed, we will consider a template galaxy cluster located at $50 \mathrm{Mpc}$, with properties representative of all nearby clusters. Our discussion, that we will keep as generic as possible, is organized as follows. In Sec. 2, we will recall the principle of the SZ calculation. In Sec. 3 we will derive the optical depths for both DM-induced and thermal electrons in an exact numerical manner and, likewise, provide analytical formulae to allow quick calculation of the SZ effect for any cluster and DM properties. In Sec. \&, we will then use these results together with a full spectral analysis to compute the signal due to the DM-induced electrons at the transition frequency where the thermal component is negligible, and show that the DM contribution to the SZ will be quite hardly observable with current and even future radio experiments. In Sec. 5, we will close the case by considering spatial diffusion effects, showing analytically that they induce a core in the electron distribution whatever the cuspiness of the DM profile. We will conclude in Sec. 6 .

\section{Principle}

There are basically two main approaches to calculate the thermal or relativistic SZ effect, the radiative transfer method proposed in e.g. [15] and early formalized in [16], and the covariant Boltzmann equation formalism [17, 18, 19, 20, 21. Recently, however, these approaches have been demonstrated to be equivalent [22, 23], which settles a self-consistent and unique framework for the SZ calculations. In the single scattering approximation, the distortion of the CMB intensity $I$ at a photon energy $E_{k}$ is given by the following equation:

$$
\Delta I_{\gamma}\left(E_{k}\right)=\tau \int_{0}^{t_{\max }} d t\left(P_{1}(t)-\delta(t-1)\right) I_{\gamma}^{0}\left(E_{k} / t\right)
$$

where $I_{\gamma}^{0}$ is the undistorted blackbody intensity, $P_{1}$ is the frequency redistribution function for a single electron-photon interaction and $t$ is the ratio of scattered to unscattered photon

\footnotetext{
${ }^{1}$ Hereafter, we will use electron for electron or positron, indifferently.
} 
energy. The integrand encodes the spectral features: the first term describes the spectral part that is shifted from energy $E_{k} / t$ to $E_{k}$, while the second term describes the part that is removed from energy $E_{k}$ to others. $\tau$, which weights the amplitude of the distortion, is the optical depth which is related to the electron density in the cluster through the line-of-sight integral

$$
\tau \equiv \sigma_{\mathrm{T}} \int d l n_{e}(\vec{x})
$$

Here $\sigma_{\mathrm{T}}$ is the non-relativistic Thomson cross section that fully characterizes interactions of CMB photons with electrons of Lorentz factor $\gamma_{e} \lesssim 10^{8}$. As far as $\tau \ll 1$, the single scattering approximation is fully justified [23].

In order to estimate the potential of a radio experiments to detect SZ signals, however, one needs to average the optical depth within the angular resolution of the apparatus,

$$
\langle\tau\rangle_{\mu_{\mathrm{res}}}=\frac{\sigma_{\mathrm{T}}}{\Delta \Omega\left(\mu_{\mathrm{res}}\right)} \int_{\Delta \Omega\left(\mu_{\mathrm{res}}\right)} d \Omega \int d l n_{e}(\vec{x})
$$

where $\mu_{\text {res }} \equiv \cos \left(\theta_{\text {res }}\right)$ characterizes the angular resolution $\theta_{\text {res }}$.

Disregarding for the moment the spectral aspects, the observation of a relativistic SZ signal on top of the thermal contribution would roughly imply that the optical depth of the additional electron component is sizable compared to the one associated with the thermal electrons. A mere comparison of the electron densities in the cluster center, as done in [10], is indicative at $0^{\text {th }}$ order. Nevertheless, because of the angular averaging, this could be misleading. Indeed, the SZ signal is in any case smeared down by angular resolution effects.

In Sec. 3, we will study the effects of this averaging procedure, which is actually very common in studies on the indirect detection of DM with $\gamma$-rays (e.g. 24]). We will consider angular resolutions between $\sim 0.1^{\prime \prime}$ and $1^{\prime}$, noticing that Planck [11] and ALMA [12, 13] could reach resolutions of $\sim 5^{\prime}$ and $\sim 1^{\prime \prime}$, respectively.

Throughout the paper, moreover, we will consider the spatial distribution of the thermal electrons to be a spherical cored isothermal:

$$
n_{e, \mathrm{th}}(r)=\frac{n_{e, \mathrm{th}}^{0}}{1+\left(\frac{r}{r_{c}}\right)^{2}},
$$

where we will fix $n_{e, \text { th }}^{0}$ to $0.01 \mathrm{~cm}^{-3}$, and $r_{c}$ to $400 \mathrm{kpc}$. These are typical values for nearby clusters that will partly characterize our template cluster.

For completeness, it is worth recalling that the SZ effect is featured by the shift of the low frequency part of the CMB spectrum towards higher frequencies, but much more marginally by that of the high frequency part towards lower frequencies. This asymmetry drives $\Delta I$ to be negative at low frequencies and positive at high frequencies. The transition frequency, at which $\Delta I=0$, is intimately related to the energy spectrum of the involved electron gas. Because the thermal and DM-induced components are found in different energy ranges, the associated transition frequencies are different. Therefore, not only do we need to compare the optical depths of both contributions, that weight the amplitudes 
of the corresponding distortions, but also to study the spectral features of $\Delta I$. For the spectral study, we will mainly focus on $\Delta I_{\chi}$ at the transition frequency of the thermal component $\left(\Delta I_{\text {th }}=0\right)$ in Sec. 1 .

\section{Injected electron density from DM annihilation or decay and optical depths}

The injection rate of relativistic electrons (and positrons) from DM annihilation or decay characterizes a nominal source term

$$
\mathcal{Q}_{n, \gamma}(E, \vec{x})=N_{0} \alpha_{n}\left(\frac{\rho_{\gamma}(\vec{x})}{m_{\chi}}\right)^{n} \mathcal{F}(E)
$$

where $N_{0}$ is the total number of electrons and positrons injected per annihilation or decay in the relevant energy range, $\mathcal{F}(E)$ is the energy spectrum normalized to unity, and $m_{\chi}$ is the DM particle mass. The index $n$ is 1 or 2 for DM decay or annihilation, respectively. Therefore, $\alpha_{1} \equiv \Gamma_{\chi}$ is a decay rate, while $\alpha_{2} \equiv \delta\langle\sigma v\rangle / 2(\delta=1$ or $1 / 2$ for Majorana or Dirac fermions, 1 for bosons) is an annihilation rate. The DM mass density profile $\rho_{\gamma}$ is indexed by its inner logarithmic slope $\gamma$, and is usually written like a spherical symmetric component with a scale radius $r_{s}$ and a scale density $\rho_{s}$ [25]

$$
\rho_{\gamma}(r)=\frac{\rho_{s}\left(r_{s} / r\right)^{\gamma}}{\left(1+\left(r / r_{s}\right)^{\alpha}\right)^{(\beta-\gamma) / \alpha}}=\rho_{s} f_{\gamma}(r) .
$$

The so-called Navarro-Frenk-White (NFW) profile [26] corresponds to $(\alpha, \beta, \gamma)=(1,3,1)$, while the Moore profile [27] is even more cuspy with $(1.5,3,1.5)$. Notice that a cored isothermal profile with $(2,2,0)$ is equivalent to the thermal electron distribution of Eq. (2.4), with $r_{s}=r_{c}$; this may be kept in mind with further benefit. We will study all of the three aforementioned profiles in the following, for both annihilating and decaying DM models. The typical values of the scale parameters found for galaxy clusters with masses $\sim 10^{15} M_{\odot}$ in cosmological N-body simulations are $r_{s} \sim 400 \mathrm{kpc}$ and $\rho_{s} \sim 0.05 \mathrm{GeV} . \mathrm{cm}^{-3}$ (e.g. [28]). We will adopt these parameters for the template cluster that we will use for our calculations throughout this paper. We summarize them in Tab. 1.

\begin{tabular}{ccccc}
\hline $\begin{array}{c}\text { distance } \\
(\mathrm{Mpc})\end{array}$ & $\begin{array}{c}R_{\mathrm{vir}} \\
(\mathrm{kpc})\end{array}$ & $\begin{array}{c}r_{s} \text { and } r_{c} \\
(\mathrm{kpc})\end{array}$ & $\begin{array}{c}\rho_{s} \\
\left(\mathrm{GeV} / \mathrm{cm}^{3}\right)\end{array}$ & $\begin{array}{c}\text { thermal } e^{-} \text {density } \\
\left(\mathrm{cm}^{-3}\right)\end{array}$ \\
50 & 2000 & 400 & 0.05 & 0.01 \\
\hline
\end{tabular}

Table 1: Properties of the template cluster used in this paper.

After their injection in the intracluster medium, relativistic electrons and positrons diffuse in space and momentum. The main processes that come into play are the energy losses, with a typical timescale $\sim 300 \mathrm{Myrs}$, and the spatial diffusion due to the scattering on the magnetic inhomogeneities. It was shown in previous analyses that since the relevant spatial diffusion scale is $\sim \mathrm{kpc}$, which is just a bit larger than typical resolution scales, 
one can neglect spatial diffusion at first order [7]. We will stick to this approximation, for which, in steady state, the diffusion equation reduces to

$$
\frac{\partial}{\partial E}\left\{b(E) \frac{d n_{e, \chi}^{n, \gamma}(E, \vec{x})}{d E}\right\}=\mathcal{Q}_{n, \gamma}(E, \vec{x})
$$

where $b(E) \equiv-d E / d t$ is the energy loss rate. This equation is easily solved:

$$
\begin{aligned}
\frac{d n_{e, \chi}^{n, \gamma}(E, \vec{x})}{d E} & =\frac{1}{b(E)} \int_{E}^{\frac{n m_{\chi}}{2}} d E_{s} \mathcal{Q}_{n, \gamma}\left(E_{s}, \vec{x}\right) \\
& =\frac{N_{0} \alpha_{n}}{b(E)}\left(\frac{\rho_{\gamma}(\vec{x})}{m_{\chi}}\right)^{n} \int_{E}^{\frac{n m_{\chi}}{2}} d E_{s} \mathcal{F}\left(E_{s}\right) .
\end{aligned}
$$

Now, to compute the optical depth, as defined in Eq. (2.2), we need to integrate this differential electron density over energy. Using the previous equation, this gives:

$$
n_{e, \chi}^{n, \gamma}(\vec{x})=N_{0} \alpha_{n}\left(\frac{\rho_{\gamma}(\vec{x})}{m_{\chi}}\right)^{n} \int_{E_{\min }}^{\frac{n m_{\chi}}{2}} \frac{d E}{b(E)} \int_{E}^{\frac{n m_{\chi}}{2}} d E_{s} \mathcal{F}\left(E_{s}\right) .
$$

We can further express the energy loss rate as $b(E)=\left(E_{0} / \tau_{\text {loss }}\right) / g(E)$, where $E_{0}=1$ $\mathrm{GeV}, \tau_{\text {loss }}$ is the typical energy loss timescale, and $g(E)$ is a dimensionless function that encodes the energy dependence of the energy loss rate. The previous equation is then more conveniently rewritten as

$$
n_{e, \chi}^{n, \gamma}(\vec{x})=N_{0} \alpha_{n}\left(\frac{\rho_{\gamma}(\vec{x})}{m_{\chi}}\right)^{n} \tau_{\text {loss }} \overline{\mathcal{F}}
$$

where we have defined

$$
\overline{\mathcal{F}} \equiv \int_{E_{\min }}^{\frac{n m_{\chi}}{2}} \frac{d E}{E_{0}} g(E) \int_{E}^{\frac{n m_{\chi}}{2}} d E_{s} \mathcal{F}\left(E_{s}\right) .
$$

Note that $\overline{\mathcal{F}} \lesssim m_{\chi} / E_{0}$ for Coulomb losses $(g(E) \propto$ cst $)$, while $\overline{\mathcal{F}} \lesssim E_{0} / E_{\text {min }}$ for inverse Compton losses $\left(g(E) \propto\left(E / E_{0}\right)^{-2}\right)$, which is in any case $\lesssim 10^{3}$.

Now, to go further in our calculation of the optical depth, we need to compute the average density of electrons within the angular resolution of the telescope.

\subsection{Line-of-sight averaged optical depth and electron density}

As stated in Sec. 2 through Eq. (2.3), the optical depth must be averaged within the experimental angular resolution. The full line-of-sight integral is not analytical in most of cases. We can reexpress Eq. (2.3) in such a way that an averaged electron density explicitly appears:

$$
\left\langle\tau_{e, \chi}^{\gamma, n}\right\rangle=2 r_{s} \sigma_{\mathrm{T}}{\widetilde{\left\langle n_{e, \chi}^{n, \gamma}\right\rangle_{\text {res }}}}
$$

Factorizing the scale radius $r_{s}$ out just translates the fact that the most important contribution to the $\mathrm{SZ}$ is expected to come from within a radius of $r_{s}$ from the cluster center 
(therefore within a distance of $2 r_{s}$ along the line of sight). This is essentially true for annihilating DM, but the equation is anyway made exact for all cases by defining the average electron density as

$$
\widetilde{\left\langle n_{e, \chi}^{n, \gamma}\right\rangle_{\text {res }}}=N_{0} \alpha_{n}\left(\frac{\rho_{s}}{m_{\chi}}\right)^{n} \tau_{\text {loss }} \overline{\mathcal{F}} \mathcal{J}^{n, \gamma}
$$

This ensures Eq. (3.8) to be an exact formulation of the angular averaging of the optical depth, provided a proper definition of the dimensionless parameter $\mathcal{J}^{n, \gamma}$, which carries the angular average:

$$
\begin{aligned}
\mathcal{J}^{n, \gamma} & \equiv \frac{1}{2 r_{s}\left(1-\mu_{\mathrm{res}}\right)} \int_{\mu_{\mathrm{res}}}^{1} d \mu \int d l\left(f_{\gamma}(r(l, \mu))\right)^{n} \\
& =\frac{1}{2 r_{s}\left(1-\mu_{\mathrm{res}}\right)} \int_{\mu_{\mathrm{res}}}^{1} d \mu 2 \int_{0}^{\sqrt{R_{\mathrm{vir}}^{2}-b^{2}}} d s\left(f_{\gamma}(r(s, b))\right)^{n} \\
& =\frac{\left(1+\mu_{\mathrm{res}}\right)}{b_{\mathrm{res}}^{2}} \int_{0}^{b_{\mathrm{res}}} \frac{d b b}{\sqrt{1-\frac{b^{2}}{D^{2}}}} \int_{0}^{\sqrt{R_{\mathrm{vir}}^{2}-b^{2}}} \frac{d s}{r_{s}}\left(f_{\gamma}\left(\sqrt{s^{2}+b^{2}}\right)\right)^{n} .
\end{aligned}
$$

$b=D \sin \left(\theta_{\text {res }}\right)$ is the impact parameter, $\mu_{\text {res }}=\cos \left(\theta_{\text {res }}\right), f_{\gamma}(r) \equiv \rho_{\gamma}(r) / \rho_{s}, s \equiv \sqrt{r^{2}-b^{2}}$ is the reduced line-of-sight variable and $R_{\text {vir }}$ is the virial radius of the cluster. We have expressed the angular integral in terms of an integral over the impact parameter, which allows a better control of numerical aspects. It is usually convenient to integrate over $b$ and $s$ with logarithmic steps, and to define a numerical cut $r_{\text {cut }}$ for $f_{\gamma}$ when $b \rightarrow 0$. Although formally set in a pure DM halo by equating the gravitational infall timescale with the annihilation timescale [29], $r_{\text {cut }}$ can be chosen as to ensure a good compromise between numerical accuracy, convergence and computing time, but should anyway obey $r_{\text {cut }} \ll b_{\text {res }}$. We remind, nevertheless, that small core radii could instead arise from other non-trivial dynamical effects involving baryons, and are actually observed at the galactic scale [30].

With the previous definitions, simple estimates of the optical depths associated with DM decay and annihilation read, respectively:

$$
\begin{aligned}
& \left\langle\tau_{e, \chi}^{1, \gamma}\right\rangle_{\text {res }}^{\text {dec }}=\tau_{0, \chi}^{\text {dec }} \frac{N_{0}}{10} \frac{\overline{\mathcal{F}}}{10^{3}} \frac{\Gamma_{\chi}}{10^{-26} \mathrm{~s}^{-1}} \frac{\tau_{\text {loss }}}{10^{17} \mathrm{~s}}\left[\frac{\rho_{s} /\left(0.05 \mathrm{GeV} / \mathrm{cm}^{3}\right)}{m_{\chi} / \mathrm{GeV}}\right]\left[\frac{2 r_{s} \mathcal{J}^{1, \gamma}}{10^{3} \mathrm{kpc}}\right] \\
& \left\langle\tau_{e, \chi}^{2, \gamma}\right\rangle_{\text {res }}^{\text {ann }}=\tau_{0, \chi}^{\text {ann }} \frac{N_{0}}{10} \frac{\overline{\mathcal{F}}}{10^{3}} \frac{\langle\sigma v\rangle}{3 \cdot 10^{-26} \mathrm{~cm}^{3} \mathrm{~s}^{-1}} \frac{\tau_{\text {loss }}}{10^{17} \mathrm{~s}}\left[\frac{\rho_{s} /\left(0.05 \mathrm{GeV} / \mathrm{cm}^{3}\right)}{m_{\chi} / \mathrm{GeV}}\right]^{2}\left[\frac{2 r_{s} \mathcal{J}^{2, \gamma}}{10^{5} \mathrm{kpc}}\right],
\end{aligned}
$$

where the typical optical depths are given by

$$
\tau_{0, \chi}^{\mathrm{dec}}=1.01 \times 10^{-6} \text { and } \tau_{0, \chi}^{\mathrm{ann}}=7.68 \times 10^{-6} .
$$

It is important to note that the values that we have taken for $N_{0}$ and $\overline{\mathcal{F}}$ typify mostly DM particles with masses above $\sim 100 \mathrm{MeV}$, since for lighter particles, the annihilation or decay will be mostly into light fermion pairs. For direct annihilation or decay in $e^{+} e^{-}$, we

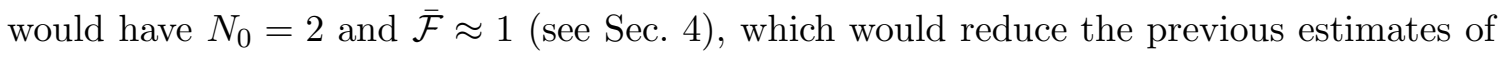
the optical depths by a factor of $5 \times 10^{3}$. 
In Fig. 1, we plot the full results. We have sketched the behaviors of the exact computation of $\widetilde{\mathcal{J}}^{n, \gamma} \equiv 2 r_{s} \mathcal{J}^{n, \gamma}$ on the one hand, and of the corresponding analytical spherical / line-of-sight approximations derived in Sec. 3.2 on the other hand, as functions of the resolution angle (or the corresponding impact parameter, which allows to deal with physical distances - see the top horizontal axis). These results are translated in terms of optical depth by means of the DM properties used in Eq. (3.11). Note that larger values of $R_{\mathrm{vir}}$ would not affect the results, since the denser part of the DM distribution is within $r_{s}$. Different values of the cluster distance $D$ would affect the scaling of horizontal axis, the closer the cluster the better the spatial resolution.

The values of the DM-induced electron optical depths derived in Eq. (3.11) have to be compared with what is expected for the thermal electrons. Because the latter obey an isothermal cored profile, as made explicit in Eq. (2.4), the function $\mathcal{J}^{1,0}$ can be used to infer their optical depth (we take advantage of that we have used $r_{c}=r_{s}$ ). We find:

$$
\langle\tau\rangle_{\text {res }}^{\text {th }}=\tau_{0, \text { th }}\left[\frac{n_{e, \text { th }}^{0}}{0.01 \mathrm{~cm}^{-3}}\right]\left[\frac{2 r_{c} \mathcal{J}^{1,0}}{10^{3} \mathrm{kpc}}\right],
$$

where the typical optical depth is found to be

$$
\tau_{0, \mathrm{th}}=2.05 \cdot 10^{-2}
$$

with our template parameters. Such a value is very close to what is actually found in dedicated studies, where $\tau \sim 10^{-3}-10^{-2}$ [14]. Moreover, this value remains a constant function of the angular resolution up to an angular size that corresponds, roughly, to the core radius $r_{c} \sim 400 \mathrm{kpc}$.

If at first glance, still disregarding the spectral aspects, we want to compare the thermal and DM optical depths, then we can use Fig. 1 (the left vertical axis gives $2 r_{s} \mathcal{J}^{n, \gamma}$ and the right vertical axis provides the translation in terms of optical depth for annihilating DM with the generic parameters used in Eq. 3.11). From this figure, we can already emphasize that unless $\langle\sigma v\rangle \gg 3 \cdot 10^{-26} \mathrm{~cm}^{3} / \mathrm{s}, \Gamma_{\chi} \gg 10^{-26} \mathrm{~s}^{-1}$ or $m_{\chi} \ll 1 \mathrm{GeV}, \gamma>1$ or $\rho_{s} \gg 0.05 \mathrm{GeV} / \mathrm{cm}^{3}$, DM decay or annihilation cannot supply a sufficient amount of electrons to compete with the thermal optical depth. This even holds for rather light DM particles in the sub-GeV mass-scale given conventional values for the other parameters. Such a statement is in agreement with [10], though based on more striking analytical calculations, but in clear disagreement with [5, 6, 7]. The detection potential of future experiments with angular resolutions $\sim 1^{\prime \prime}$ seems therefore very weak except for light DM with $m_{\chi}<1 \mathrm{GeV}$ either associated with large annihilation or decay rates, or distributed with very cuspy profiles $(\gamma \gtrsim 1.5)$. This latter feature is, however, strongly disfavored by most of the recent cosmological N-body simulations (e.g. [31]).

Before tackling the full spectral analysis, we derive hereafter some approximated analytical expressions of the line-of-sight integral $\mathcal{J}^{n, \gamma}$. This set of fast formulae is aimed at providing some quick estimates of the optical depth for any annihilating or decaying DM model, and for any density profile discussed below Eq. (3.2). 


\subsection{Analytical approximations}

The relevant part of the DM distribution is that within the angular resolution of the telescope. The current and foreseen angular resolutions are not better than $\theta_{\text {res }} \sim 1^{\prime \prime}$. Since nearby clusters lie at distances around $50 \mathrm{Mpc}$, the spatial resolution can hardly be better than $b_{\text {res }} \sim D \theta_{\text {res }} \sim 0.1 \mathrm{kpc}$ ( $b$ is defined as the impact parameter). Such values are much smaller than the typical scale radii $r_{s}$ found for cluster density profiles, so we can approximate the density profile of Eq. (3.2) as

$$
\rho_{\gamma}(r) \approx \rho_{s}\left(\frac{r_{s}}{r}\right)^{\gamma} \Leftrightarrow f_{\gamma}(r) \approx\left(\frac{r_{s}}{r}\right)^{\gamma} .
$$

Beside the fact that the impact parameter is, in most cases, smaller than the scale radius $r_{s}$, this approximation is further justified from that the dominant part of the line-of-sight DM contribution arises within a sphere of radius $r_{s}$ by the cluster center (the argument is weaker for decaying DM). We will therefore disregard the whole spatial extent of the cluster characterized by $R_{\mathrm{vir}}$, and focus only on the region within $r_{s}$. We further assume $D \gg r_{s} \gg b_{\text {res }} \gg r_{\text {cut }}$. This latter cut-off radius $r_{\text {cut }}$ has already been discussed below Eq. (3.10). It has no impact except for diverging injection rates, scaling like $r^{-\alpha}$ with $\alpha \geq 3$ (corresponding for instance to annihilating DM distributed with a Moore profile); we will use $r_{\text {cut }}=10^{-3} \mathrm{pc}$ in the following. We will develop two types of approximations, one based on a spherical average, the other based on a more accurate line-of-sight treatment.

\subsubsection{The spherical approximation}

If the impact parameter is sufficiently small, the average value of the electron density determined in Eq. (3.9) can be approximated by a spherical integral of $n_{e, \chi}$ around $b_{\text {res }}$, weighted by the conic volume $\sim \pi b_{\text {res }}^{2} R_{\text {vir }} / 3$ of the cluster roughly carried by the telescope resolution. In this case, we can derive a spherical approximation of the dimensionless function $\mathcal{J}^{n, \gamma}$ as follows

$$
\mathcal{J}^{n, \gamma} \approx \mathcal{J}_{\mathrm{sph}}^{n \gamma} \approx \frac{12}{b_{\mathrm{res}}^{2} R_{\mathrm{vir}}} \frac{R_{n, \beta, \gamma}}{r_{s}} r_{s}^{n \gamma}\left\{\begin{array}{l}
\left.\frac{r^{3-n \gamma}}{3-n \gamma}\right|_{r_{\text {rut }}} ^{b_{\text {cut }}}, n \gamma \neq 3 \\
\ln \left(\frac{b_{\text {res }}}{r_{\text {cut }}}\right) \text { otherwise }
\end{array}\right.
$$

A given product of $n \gamma$ corresponds to one or two DM configurations, as recalled in Tab. 2 . The scaling with $b_{\text {res }}$ is found to match exactly the actual behavior of $\mathcal{J}^{n, \gamma}$, but we need

to define and tune a new scale, $R_{n, \beta, \gamma}$, to get closer to the actual value of the optical depth ( $\beta=3$ for the NFW and Moore profiles, and $\beta=2$ for cored isothermal profiles). $R_{n, \beta, \gamma}$ should be of the order $r_{s}$ for consistency reasons (see Eqs. 3.8 and 3.9). We actually find that

$$
R_{n, \beta, \gamma}=r_{n, \gamma}^{0}\left(\frac{b_{\mathrm{res}}}{r_{n, \gamma}}\right)^{\beta-3}
$$

with

$$
r_{1, \gamma}^{0}=r_{s} ; r_{1, \gamma}=\frac{r_{s}}{7} \text { and } r_{2, \gamma}^{0}=r_{2, \gamma}=r_{s}
$$


provides a reasonable approximation of the accurate numerical results, by less than one order of magnitude for cluster parameters not too far from those of our template model. This is therefore consistent with the values expected for $R_{n, \beta, \gamma}$. The comparison with the accurate result is shown in Fig. 1. Although this approximation allows to derive orders of magnitude quite quickly, we recall that it is very simplistic, so one should use it with care. We recommend not to use it for very detailed analyses, but instead to make the full computation of Eq. (3.8). The line-of-sight approximation that we propose in the next paragraph turns out to be much more accurate; still, the spherical case is rather intuitive and provides complementary insights.

\subsubsection{The line-of-sight approximation}

With the same approximation as above (see Eq. 3.15), one can also try to find analytical solutions to the line-of-sight integral of Eq. (3.10). Assuming that the impact parameter $b \ll r_{s} \ll \ll D$, which is always the case for typical angular resolutions, we can write $\mathcal{J}^{n, \gamma} \approx \mathcal{J}_{\text {los }}^{n \gamma}$, where:

$$
\mathcal{J}_{\mathrm{los}}^{n \gamma} \equiv \frac{\left(1-\mu_{\mathrm{res}}\right)}{b_{\mathrm{res}}^{2}} \int_{0}^{b_{\mathrm{res}}} d b b \int_{0}^{r_{s}} \frac{d s}{r_{s}}\left(\frac{r_{s}^{2}}{s^{2}+b^{2}}\right)^{n \gamma / 2} .
$$

The upper bound of the integral over $s$ is $r_{s}$ and not $R_{\text {vir }}$ to be consistent with the validity domain of our current approximations. The different possible combinations of $n \gamma$ are recalled in Tab. 2 .

\begin{tabular}{cccc}
\hline nature / profile & $\begin{array}{c}\text { cored } \\
(\gamma=0)\end{array}$ & $\begin{array}{c}\text { NFW } \\
(\gamma=1)\end{array}$ & $\begin{array}{c}\text { Moore } \\
(\gamma=3 / 2)\end{array}$ \\
decaying $(n=1)$ & 0 & 1 & $3 / 2$ \\
annihilating $(n=2)$ & 0 & 2 & 3 \\
\hline
\end{tabular}

Table 2: Various combinations of the product $n \gamma$ and associated DM configurations for the analytical functions $\mathcal{J}_{\mathrm{sph} \text {. }}^{n \gamma}$ and $\mathcal{J}_{\text {los }}^{n \gamma}$ (see Secs. 3.2.1 and 3.2.2).

The function $\mathcal{J}_{\text {los }}^{n \gamma}$ can be computed analytically in the following cases:

$$
\begin{aligned}
& \mathcal{J}_{\text {los }}^{0}=\frac{\left(1+\mu_{\text {res }}\right)}{2} \\
& \mathcal{J}_{\text {los }}^{1}=\frac{\left(1+\mu_{\text {res }}\right)}{2} \ln \left(\frac{2 r_{s}}{b_{\text {res }}}\right) \\
& \mathcal{J}_{\text {los }}^{2}=\frac{\left(1+\mu_{\text {res }}\right) \pi}{2} \frac{r_{s}}{b_{\text {res }}} \\
& \mathcal{J}_{\text {los }}^{3}=\left(1+\mu_{\text {res }}\right)\left(\frac{r_{s}}{b_{\text {res }}}\right)^{2} \ln \left(\frac{b_{\text {res }}}{(1+\sqrt{2}) r_{\text {cut }}}\right) .
\end{aligned}
$$

Armed with these equations, the approximated optical depths are merely proportional to $2 r_{s} \mathcal{J}_{\text {los }}^{n \gamma}$, as given in Eqs. (3.8) and (3.9). The results are also reported in Fig. 1, where the agreement with the exact calculation is shown to be quite good. 


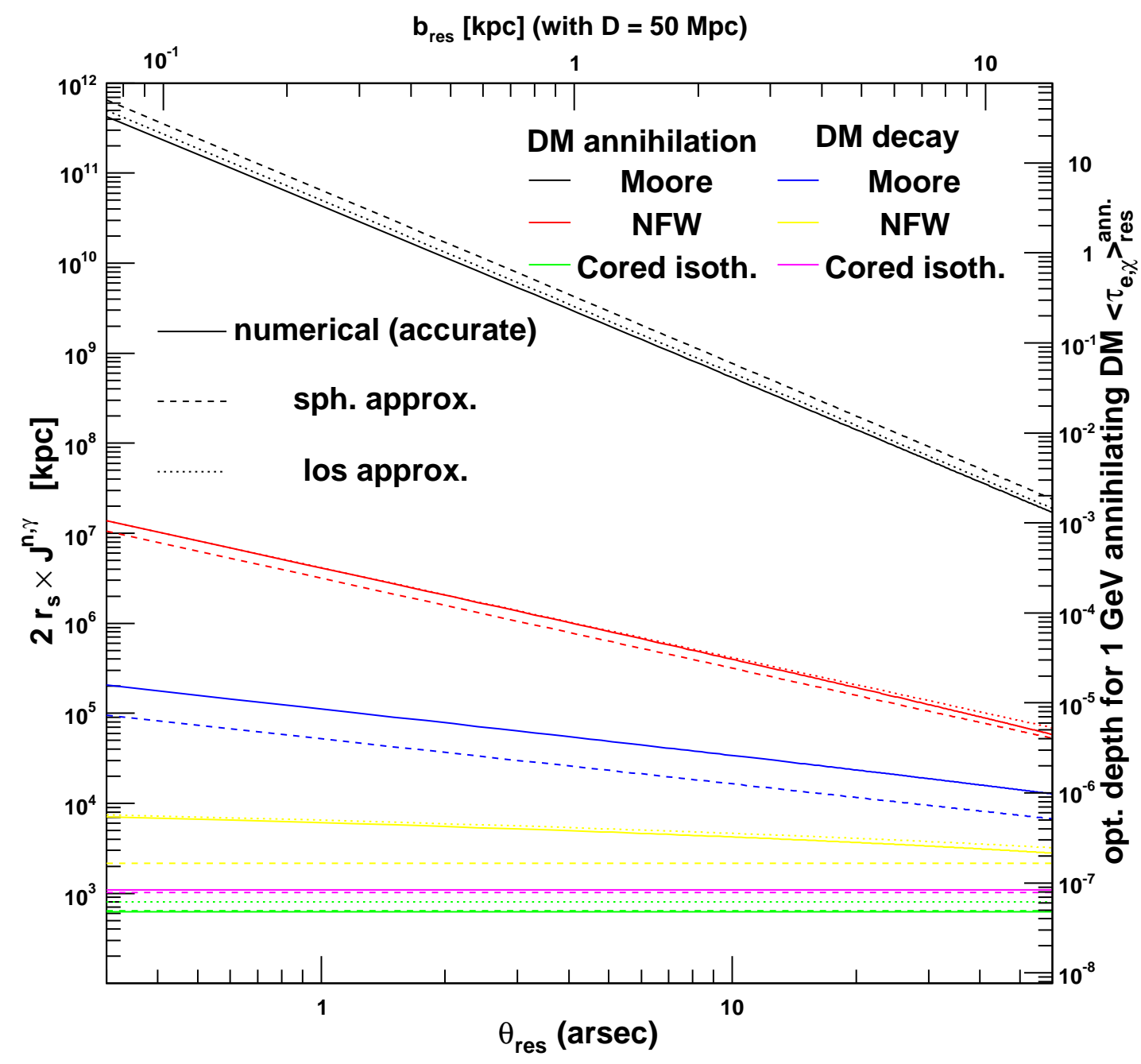

Figure 1: Line-of-sight integral for the accurate numerical computation, the spherical approximation and the so-called line-of-sight approximation, as a function of the angular resolution over which it is averaged. The bottom horizontal axis is the angular resolution, the top vertical one is the corresponding impact parameter $b_{\text {res }}=D \sin \left(\theta_{\text {res }}\right)$. The left vertical axis is $2 r_{s} \mathcal{J}^{n, \gamma}$ - see Sec. 3.1 — while the right vertical axis translates those values in terms of optical depth for typical DM parameters, but only for annihilating DM (the optical depth axis for decaying DM would be a factor of $\sim 1.5$ smaller, for nominal parameters - see Eq. 3.11).

\subsection{Thermal to DM optical depth ratio $\eta_{\chi}$}

To size the relative amplitude of the SZ distortion induced by DM annihilation or decay on top of the thermal component, it is useful to derive the ratio $\eta_{\chi} \equiv\left\langle\tau_{e, \chi}\right\rangle_{\text {res }} /\left\langle\tau_{e, \text { th }}\right\rangle_{\text {res }} \gtrsim 0.1$, at any frequency where $\Delta I_{\mathrm{th}} \neq 0$. Since the frequency where $\Delta I$ crosses 0 is different for each electron component, it is further important to compare the absolute amplitude of $\Delta I_{\chi}$, at the frequency where $\Delta I_{\text {th }}=0$, with the current experimental sensitivities, i.e. 
$\Delta I / I \sim 10^{-6}$. We will estimate the ratio $\eta_{\chi}$ below, but postpone our discussion of the latter point in Sec. 6 . For the sake of clarity, we will base the following calculation on the assumption that the DM density profile is an NFW $(\gamma=1)$. Likewise, we will use the analytical line-of-sight approximation derived in the previous section, which turns out to be the most precise.

Using Eqs. (3.11) and (3.13), the ratio of the DM to thermal optical depths is proportional to $\mathcal{J}_{\text {los }}^{1(2)} / \mathcal{J}_{\text {los }}^{0}$, for decaying (annihilating) DM. More explicitly, we get, for decaying DM,

$$
\begin{aligned}
\eta_{\chi}^{\mathrm{dec}} \equiv & \frac{\left\langle\tau_{e, \chi}^{1,1}\right\rangle_{\mathrm{res}}^{\mathrm{dec}}}{\left\langle\tau_{e, \mathrm{th}}\right\rangle_{\mathrm{res}}} \\
= & \frac{\tau_{0, \chi}^{\mathrm{dec}}}{\tau_{0, \mathrm{th}}}\left[\frac{n_{e, \mathrm{th}}^{0}}{0.01 \mathrm{~cm}^{-3}}\right]^{-1} \frac{N_{0}}{10} \frac{\overline{\mathcal{F}}}{10^{3}} \frac{\Gamma_{\chi}}{10^{-26 \mathrm{~s}^{-1}}} \frac{\tau_{\text {loss }}}{10^{17_{\mathrm{s}}}}\left[\frac{\rho_{s} /\left(0.05 \mathrm{GeV} / \mathrm{cm}^{3}\right)}{m_{\chi} / \mathrm{GeV}}\right]\left[\frac{\mathcal{J}_{\text {los }}^{1}}{\mathcal{J}_{\text {los }}^{0}}\right] \\
= & \eta_{\chi}^{0, \text { dec }}\left[\frac{n_{e, \mathrm{th}}^{0}}{0.01 \mathrm{~cm}^{-3}}\right]^{-1} \frac{N_{0}}{10} \frac{\overline{\mathcal{F}}}{10^{3}} \frac{\Gamma_{\chi}}{10^{-26} \mathrm{~s}^{-1}} \frac{\tau_{\text {loss }}}{10^{17} \mathrm{~s}}\left[\frac{\rho_{s} /\left(0.05 \mathrm{GeV} / \mathrm{cm}^{3}\right)}{m_{\chi} / \mathrm{GeV}}\right] \times \\
& \ln \left[\frac{2\left(r_{s} / 400 \mathrm{kpc}\right)}{b_{\mathrm{res}} / 1 \mathrm{kpc}}\right]
\end{aligned}
$$

with

$$
\eta_{\chi}^{0, \mathrm{dec}}=3.29 \times 10^{-4}
$$

For annihilating DM, the result is proportional to $\mathcal{J}_{\text {los }}^{2} / \mathcal{J}_{\text {los }}^{0}$, and we have instead

$$
\begin{aligned}
\eta_{\chi}^{\text {ann }} \equiv & \frac{\left\langle\tau_{e, \chi}^{1,1}\right\rangle_{\text {res }}^{\text {ann }}}{\left\langle\tau_{e, \text { th }}\right\rangle_{\text {res }}} \\
= & \eta_{\chi}^{0, \text { ann }}\left[\frac{n_{e, \text { th }}^{0}}{0.01 \mathrm{~cm}^{-3}}\right]^{-1} \frac{N_{0}}{10} \frac{\overline{\mathcal{F}}}{10^{3}} \frac{\langle\sigma v\rangle}{3 \cdot 10^{-26} \mathrm{~cm}^{3} \mathrm{~s}^{-1}} \frac{\tau_{\text {loss }}}{10^{17} \mathrm{~s}}\left[\frac{\rho_{s} /\left(0.05 \mathrm{GeV} / \mathrm{cm}^{3}\right)}{m_{\chi} / \mathrm{GeV}}\right]^{2} \times \\
& {\left[\frac{r_{s} / 400 \mathrm{kpc}}{b_{\mathrm{res}} / 1 \mathrm{kpc}}\right], }
\end{aligned}
$$

where

$$
\eta_{\chi}^{0, \text { ann }}=4.71 \times 10^{-3}
$$

The relevant parameters here are $\eta_{\chi}^{0, \text { dec }}$ and $\eta_{\chi}^{0, \text { ann }}$, which both are $\ll 0.1$ for most of DM models and for current and future experimental performances. Because the optical depth sizes the amplitude of the spectral distortion (see Eq. 2.1), this means that detecting a SZ signal from DM annihilation or decay on top of the thermal contribution demands very strong spectral distortions of the former with respect to the latter. We study the spectral features of the SZ signatures in the next section.

\section{Spectral distortion analysis}

To complete our study, we have to tackle a full spectral analysis. Nevertheless, what is quantitatively interesting is the amplitude of the spectral change due to DM at the SZ 
transition frequency of the thermal gas, i.e. when its global effect is null. In this section, therefore, we estimate the amplitude of $\Delta I_{\chi}$ at the frequency for which $\Delta I_{\text {th }}=0$, to size the potential impact of DM at the transition frequency of the thermal background. We adopt the formalism developed in [32], which is very well suited for our calculation. We refer the reader to that article for more details on the quantities and results that are used below.

Let us first define the reduced blackbody intensity $i(x) \equiv I\left(E_{k}\right) / i_{0}=x^{3} /\left(e^{x}-1\right)$, where $x \equiv E_{k} /\left(k T_{0}\right), T_{0}$ is the CMB temperature, and $i_{0} \equiv 2\left(k T_{0}\right)^{3} /(h c)^{2}$. With these notations, Eq. (2.1) can be rewritten in terms of the reduced intensities

$$
\delta i(x)=\tau(j(x)-i(x))
$$

where we further define the scattered photon intensity as:

$$
j(x) \equiv \int_{0}^{t_{\max }} d t P_{1}(t) i(x / t) .
$$

The photon frequency redistribution function $P_{1}(t)$, valid in the single scattering approximation, depends on the normalized injected electron spectrum $\mathcal{F}(E)=\mathcal{F}(\bar{p})(\bar{p} \equiv$ $p /\left(m_{e} c\right)=\gamma_{e} \beta_{e}=\sqrt{\gamma_{e}^{2}-1}$ being the reduced electron momentum) as follows:

$$
P_{1}(t)=\int d \bar{p} \mathcal{F}(\bar{p}) P_{1}(t, \bar{p})
$$

where $P_{1}(t, \bar{p})$ has the following analytical form

$$
\begin{gathered}
P_{1}(t, \bar{p})=-\frac{3|1-t|}{32 \bar{p}^{6} t}\left\{1+\left(10+8 \bar{p}^{2}+4 \bar{p}^{4}\right) t+t^{2}\right\}+ \\
\frac{3(1+t)}{8 \bar{p}^{5}}\left\{\frac{3+3 \bar{p}^{2}+\bar{p}^{4}}{\sqrt{1+\bar{p}^{2}}}-\frac{3+2 \bar{p}^{2}}{\bar{p}}\left(\operatorname{asinh}(\bar{p})-\frac{|\ln (t)|}{2}\right)\right\} .
\end{gathered}
$$

The maximal frequency shift, above which $P_{1}(t, \bar{p})=0$, fulfills the condition $\left|\ln \left(t_{\max }\right)\right|=$ $2 \operatorname{asinh}(\bar{p})$.

Armed with these equations, we can derive the intensity shift $\delta i$ for any electron component, given its spectrum. For the thermal electrons, we will use a Maxwell-Boltzmann spectrum

$$
\mathcal{F}_{\mathrm{th}}(\bar{p})=\frac{\beta_{\mathrm{th}}}{K_{2}\left(\beta_{\mathrm{th}}\right)} \bar{p}^{2} \exp \left(-\beta_{\mathrm{th}} \sqrt{1+\bar{p}^{2}}\right)
$$

where $\beta_{\mathrm{th}}=m_{e} c^{2} /\left(k T_{\mathrm{th}}\right)$ is the inverse reduced electron temperature, and where the normalization is ensured with the modified Bessel function of the second kind $K_{2}$. The resulting redistribution function (see Eq. 4.3) is shown in Fig. 2, for different temperatures.

For the DM electron yield, we will assume that the injected spectrum is $\propto \delta(E-$ $\left.n m_{\chi} / 2\right)$, with $n=1$ or 2 for decay or annihilation, respectively. This is equivalent to assuming a direct annihilation or decay in $e^{+} e^{-}$, which is particularly relevant for light 


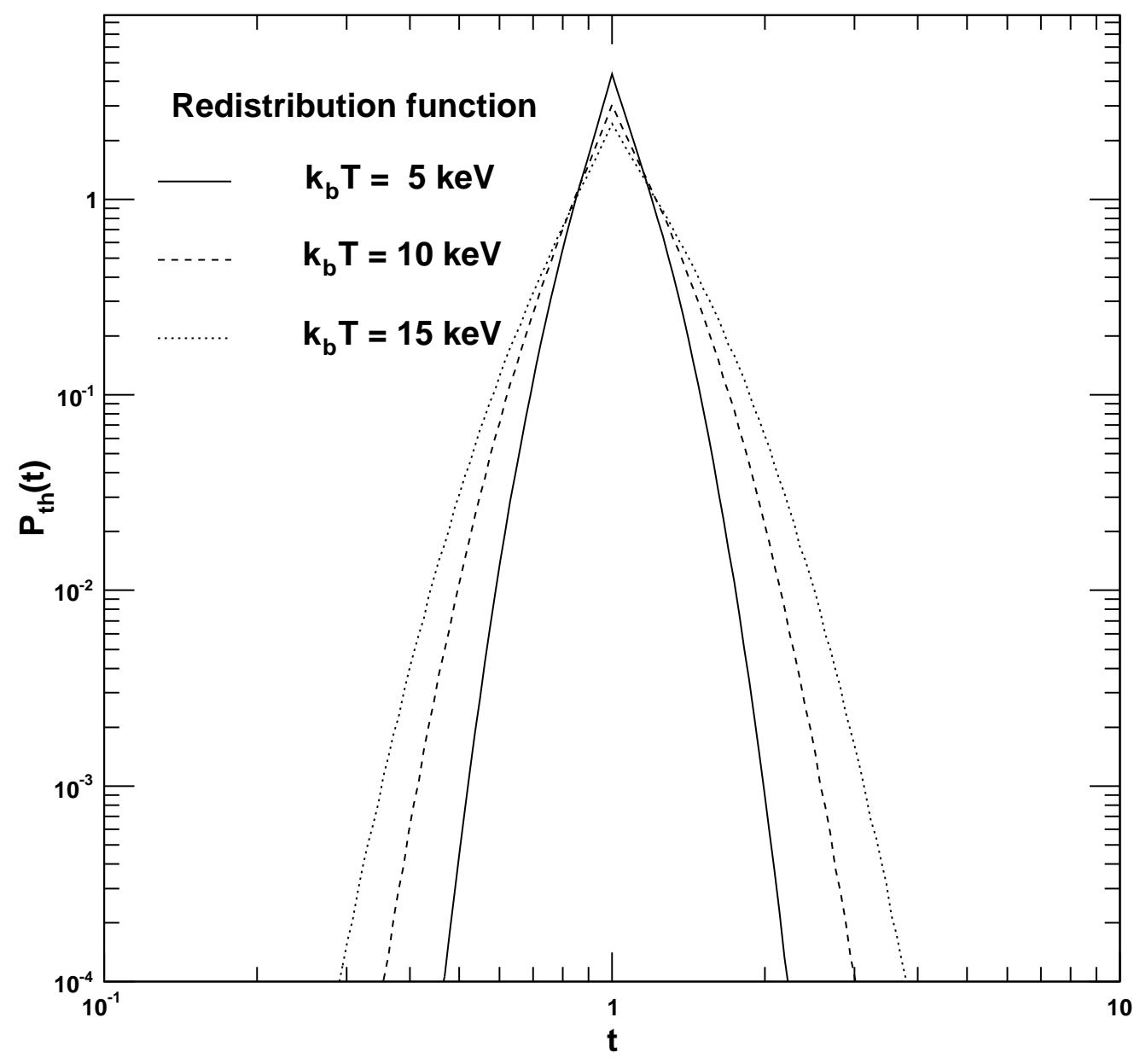

Figure 2: Frequency redistribution function for different thermal electron gas temperatures, $k T_{\text {th }}=$ $5,10,15 \mathrm{keV}$.

DM particles but a very optimistic spectral hypothesis in the general case. We can thus determine the normalized equilibrium spectrum with Eq. (3.5):

$$
\mathcal{F}(E)=\frac{K}{b(E)} \quad\left(\text { for } E \leq n m_{\chi} / 2\right)
$$

where $K=K\left(E_{\min }, E_{\max }\right)$ is a normalization constant such that $\int d E \mathcal{F}(E)=1$ in the energy range of interest. For the energy loss rate $b(E)$, we can combine the two most relevant regimes, i.e. the Coulomb losses below $E_{0}=1 \mathrm{GeV}$, of timescale $\tau_{\text {Coul. }}$ and the 


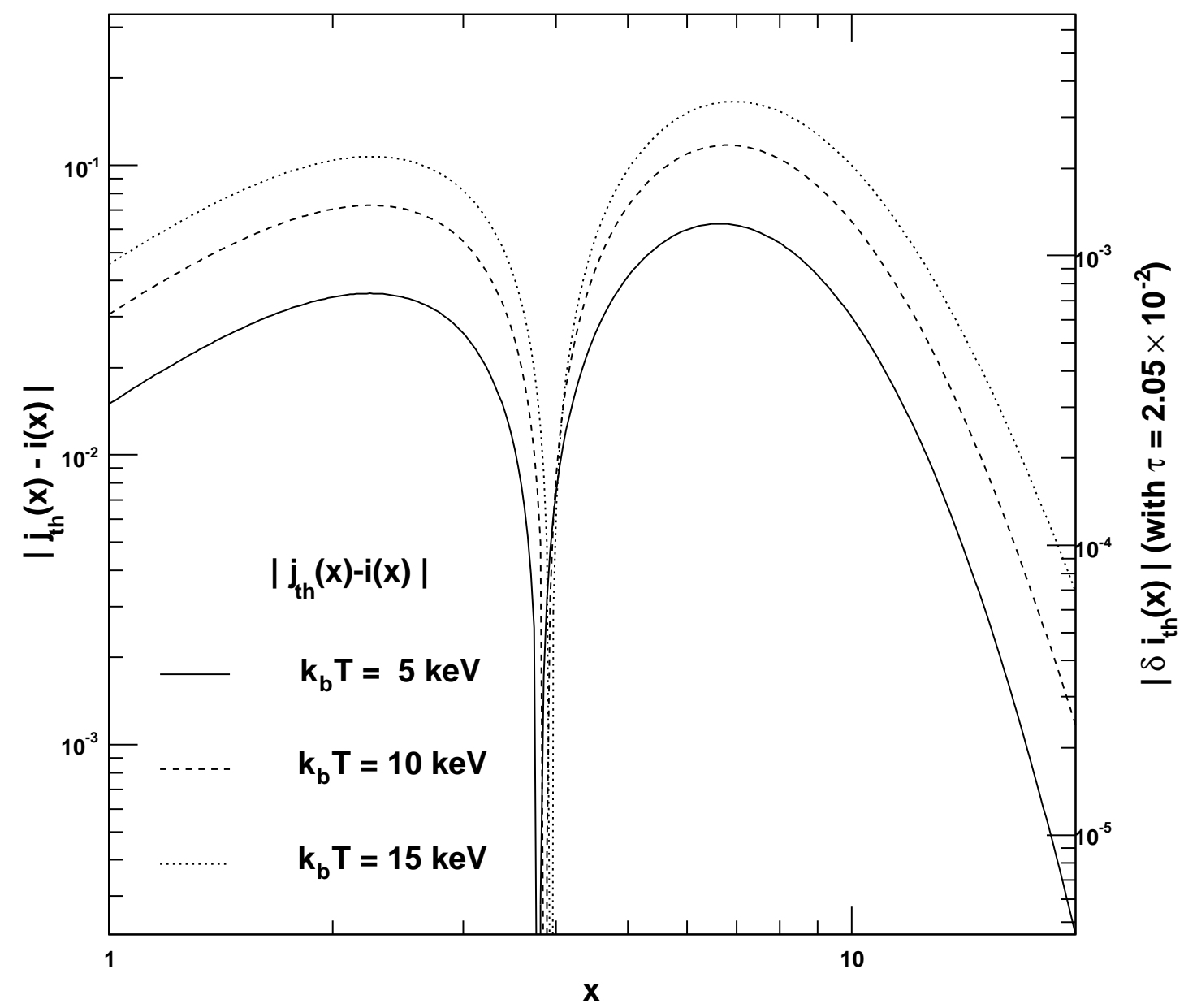

Figure 3: $\delta i_{\text {th }}(x) / \tau$ for different thermal electron gas temperatures, $k T_{\text {th }}=5,10,15 \mathrm{keV}$ (the right vertical axis gives $\left|\delta i_{\mathrm{th}}(x)\right|$ for our template cluster, with an optical depth of $\tau=2.05 \times 10^{-2}$ ).

inverse Compton losses on CMB above [33], of timescale $\tau_{\text {IC }}$. Actually, we have:

$$
\begin{aligned}
\frac{b(E)}{10^{-16} \mathrm{GeV} / \mathrm{s}} & =b_{\mathrm{IC}}(E)+b_{\text {Coul. }}(E) \\
& =0.265\left(\frac{E}{E_{0}}\right)^{2}+\frac{6.20}{\beta_{e}} \frac{n_{e, \mathrm{th}}}{1 \mathrm{~cm}^{-3}}\left\{1+\ln \left(\frac{E / E_{0}}{n_{e, \mathrm{th}} / 1 \mathrm{~cm}^{-3}}\right)\right\},
\end{aligned}
$$

where the Coulomb loss rate is taken from [34. We can simplify the previous expression 
by means of the timescales

$$
\begin{aligned}
b(E) & \simeq \frac{E_{0}}{\tau_{\text {Coul. }}}+\frac{E^{2}}{E_{0} \tau_{\text {IC }}}=\frac{E_{0}}{\tau_{\text {Coul. }}}\left\{\alpha_{\tau}\left(\frac{E}{E_{0}}\right)^{2}+1\right\} \\
\frac{\tau_{\text {Coul. }}}{10^{16} \mathrm{~s}} & \equiv 0.16 \times \frac{\beta_{e}}{n_{e, \text { th }} / 1 \mathrm{~cm}^{-3}} ; \quad \frac{\tau_{\text {IC }}}{10^{16} \mathrm{~s}} \equiv 3.77 \text { and } \alpha_{\tau} \equiv \frac{\tau_{\text {Coul. }}}{\tau_{\text {IC }}} .
\end{aligned}
$$

With this form of energy loss rate, we can reexpress Eq. (4.6) as

$$
\mathcal{F}(E)=\frac{K\left(E_{\min }, m_{\chi}\right) \tau_{\text {coul }}}{\alpha_{\tau} \frac{E^{2}}{E_{0}^{2}}+1} .
$$

The correspondence between energy and reduced momentum is straightforward: $\bar{p}=$ $\sqrt{\gamma_{e}^{2}-1}$, and $d E=\bar{p} m_{e}^{2} c^{4} d \bar{p} / E$. The energy lower bound $E_{\min }$ must be consistent with the cluster age, DM-induced electrons having essentially been injected since the cluster has formed. Therefore, these electrons had only a time of the order of the cluster age $\Delta t \approx t_{\mathrm{cl}}$ to lose their energy. We can compute the minimal energy $E_{\min } \geq m_{e} c^{2}$ corresponding to any injected energy $E$ by demanding $\int_{E_{\min }}^{E} d E^{\prime} \frac{E_{0}}{b\left(E^{\prime}\right)} \leq \Delta t$, which is equivalent to $K^{-1}\left(E_{\min }, E\right) \leq \Delta t$. For typical cluster formation redshifts around $5, E_{\min }$ is merely found to be $m_{e} c^{2}$.

The photon redistribution function obtained from the DM-induced electrons, once the normalized spectrum defined in Eq. (4.6) has been injected into Eq. (4.3), is shown in Fig. 田, for different WIMP masses, assuming direct annihilation or decay in $e^{+} e^{-}$.

Notice that when coming to the numerical calculation of the optical depth, we will take advantage of the estimates performed in the previous section by remarking that the function $\overline{\mathcal{F}}$ defined in Eq. (3.7) obeys rigorously $\tau_{\text {loss }} \overline{\mathcal{F}}=K^{-1}\left(E_{\min }, m_{\chi}\right)$, in the current DM configuration. If we assume that $\tau_{\text {loss }} \approx \tau_{\text {coul }} \approx \tau_{\text {ic }}$, then we have $\overline{\mathcal{F}} \approx 1$. Hence, with such an assumption, we can rescale the optical depths given in Eq. (3.12) accordingly.

Our aim is to size $\delta i_{\chi}\left(x_{\mathrm{th}}\right)$ at a frequency $x_{\mathrm{th}}$ such that $\delta i_{\mathrm{th}}\left(x_{\mathrm{th}}\right)=0 . x_{\mathrm{th}}$ is readily found by computing Eq. (4.1), given the thermal electron temperature. For completeness, we take three different temperatures, $k T_{\text {th }}=5,10,15 \mathrm{keV}$, though it is well known that the transition frequency is much less dependent on the temperature than the amplitude, as shown in Fig. 3. As illustrated in that plot for the three cases, we find $x_{\mathrm{th}} \simeq 4$. We can now calculate

$$
\delta i_{\chi}\left(x_{\mathrm{th}}\right)=\tau_{\chi}\left(j_{\chi}\left(x_{\mathrm{th}}\right)-i\left(x_{\mathrm{th}}\right)\right)
$$

with

$$
j_{\chi}\left(x_{\mathrm{th}}\right)=\int_{0}^{t_{\max }} d t P_{1}\left(t, \bar{p}_{\chi}\right) i(x / t),
$$

and for different DM particle masses.

We first report the behavior obtained for $\delta i_{\chi}(x) / \tau_{\chi}$ with different WIMP masses in Fig. 5, assuming direct annihilation in $e^{+} e^{-}$(also valid for decaying DM, but corresponding then to twice heavier WIMP masses). The factor of $1 / \tau_{\chi}$ allows a prediction independent 


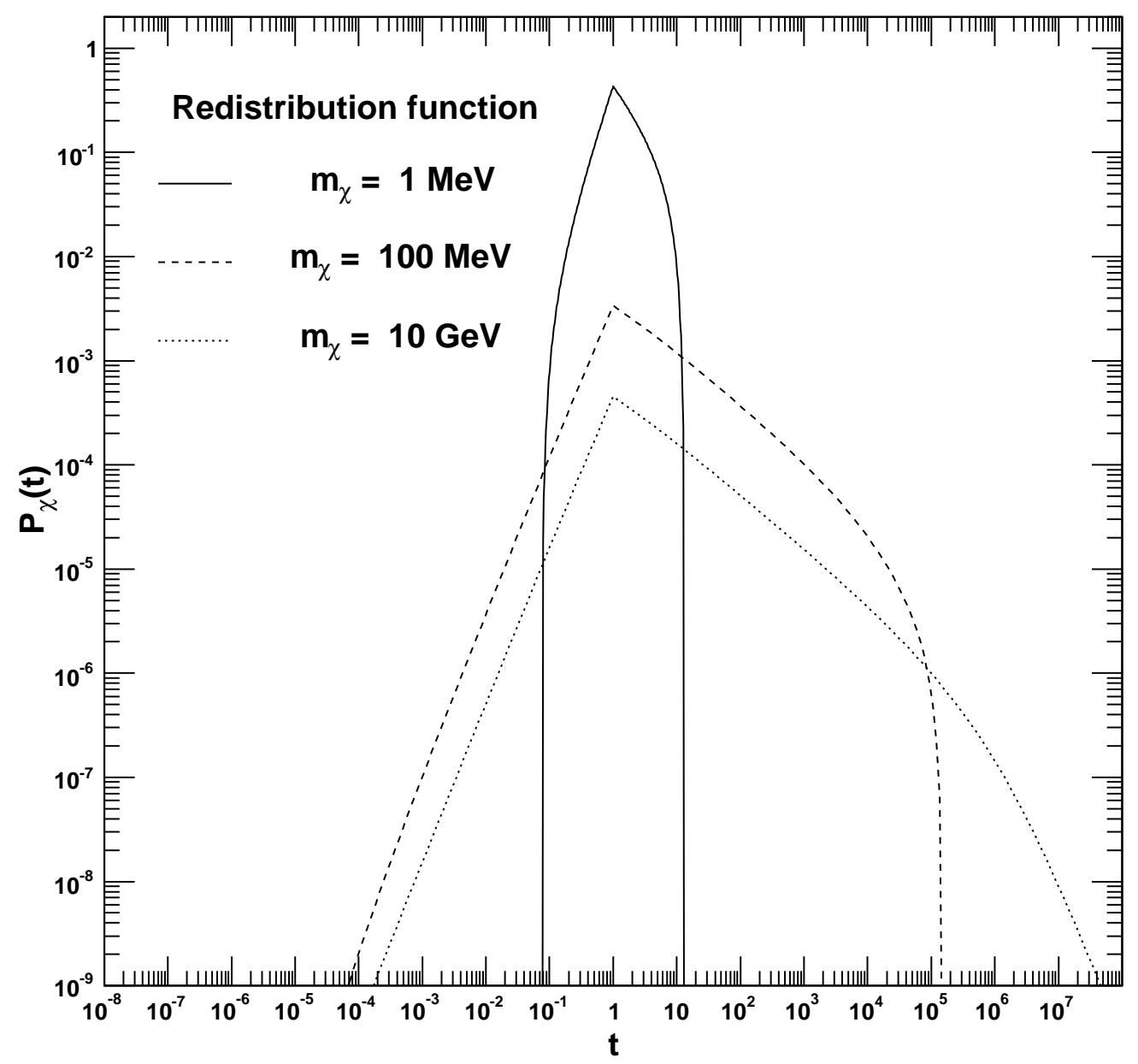

Figure 4: Redistribution functions for different WIMP masses, assuming $\chi \chi \rightarrow e^{+} e^{-}$. Decaying DM functions are identical for $\chi \rightarrow e^{+} e^{-}$but with twice the indicated masses.

of the electron density, i.e. independent of the cluster halo parameters. The right vertical axis gives the conversion in terms of $\delta i_{\chi}(x)$, assuming $\tau_{\chi}=1.5 \times 10^{-9}$ (see below), which reaches a maximum of $\delta i \sim 10^{-10}$ around the transition frequency of the thermal component $x_{\text {th }} \sim 4$. This maximal value corresponds to an annihilating DM of $1 \mathrm{GeV}$ distributed with an NFW profile and observed from our template cluster within an angular resolution of 10" (see Eq. 3.11 and Fig. 11). It is anyway easily rescaled for other configurations.

In Fig. 6, we also trace $\delta i_{\chi}\left(x_{\mathrm{th}}\right) /\left(\tau_{\chi} m_{\chi}^{n} i_{0}\left(x_{\mathrm{th}}\right)\right)$, i.e. at the transition frequency of the thermal component, as a function of the DM mass $m_{\chi} ; n=1$ (2) for decaying (annihilating) DM. We implement the expected mass dependence of the optical depth thanks to the factor of $1 / m_{\chi}^{n}$. To compare with experimental sensitivities, we have converted our result in terms 
of the relative intensity shift $\delta i$, but only in the case of DM annihilation ( $c f$. the right vertical axis). To do so, we have used the angular average of $\tau_{\chi}$ as derived in Eq. (3.11), which rests on the template DM halo defined in Tab. 1. Likewise, to be consistent with the assumption of annihilation or decay in $e^{+} e^{-}$, however, we have used $N_{0}=2$, and, as discussed above, $\overline{\mathcal{F}}=1$ (i.e. for $\tau_{0, \chi}$, we have taken the values of Eq. 3.12 divided by $5 \cdot 10^{3}$, which gives $\left.\tau_{0, \chi}=1.5 \times 10^{-9}\right)$. To switch to the $\delta i$ associated with DM decay, one may apply a factor of $1.01 / 7.68 \approx 0.13$ to the right vertical scale (see Eq. 3.11). Notice that the scaling with $m_{\chi}$ is trivial, $\propto 1 / m_{\chi}{ }^{2}$ for DM annihilation, and $\propto 1 / m_{\chi}$ for DM decay.

From the results presented in Figs. 5 and 6, it seems quite difficult to detect any SZ signal from DM annihilation or decay, since the typical values obtained for the relative intensity shift is $\delta i_{\chi} \sim 10^{-10}$, still far from current experimental sensitivities (Planck was optimized for $\delta i \sim 10^{-6}$ [11], i.e. the $\mu \mathrm{K}$ level in terms of temperature). However, we remind that this particular value of the intensity shift is connected to some assumptions. For the line-of-sight integral, which depends on the cluster halo properties, we have used $2 r_{s} \mathcal{J}=10^{3}$ and $10^{5} \mathrm{kpc}$ for DM decay and annihilation, respectively, which corresponds to observing our NFW template cluster within an angular resolution of $\sim 10^{\prime \prime}$ (see Fig. 1). Moreover, there are additional assumptions on $m_{\chi} \sim 1 \mathrm{GeV}$ and on the DM properties, though quite generic (see Eq. 3.11). Anyway, we emphasize that these assumptions are rather optimistic as regards the DM modeling, and should therefore be considered as conservative. For comparison, the intensity shift is shown to be much larger for thermal electrons (see Fig. 3 with the vertical right axis), $\delta i \sim 10^{-4}-10^{-3}$, over a broad band of frequencies, except at the transition frequency. Hence, the DM effect is likely too small in the major part of the "natural" DM parameter space, but might be still sizable for very light DM candidates and very cuspy profiles.

To figure out the influence of both the DM halo cuspiness and the experimental angular resolution, one can read off the left vertical axis of Fig. 1, which provides values of the lineof-sight integral for different cluster halo profiles as functions of the angular resolution. To boost our predictions by $\gtrsim 5$ orders of magnitude we would have to consider very cuspy profiles $(\gamma \gtrsim 1.5)$ and in the meantime very deep spatial resolutions ( $b_{\text {res }} \sim 0.1 \mathrm{kpc}$, or equivalently $\theta_{\text {res }} \sim 1^{\prime \prime}$ ), at least for the template set of halo parameters of Tab. 1. We will show in Sec. 5, however, that even this over-optimistic case is actually limited by spatial diffusion effects.

If we stick to an NFW profile, an alternative to enhance our predictions would be to consider very light annihilating WIMPs, in the sub-GeV mass-scale. However, in that case, the value of the annihilation cross considered in Eq. (3.11) is bounded by astrophysical observations in the Milky Way. Indeed, it was found in [35, 36], that the annihilation cross section for light DM must obey $\langle\sigma v\rangle \lesssim 10^{-31}\left(m_{\chi} / \mathrm{MeV}\right)^{2} \mathrm{~cm}^{3} \mathrm{~s}^{-1}$ to not overshoot the 511 $\mathrm{keV}$ bulge emission measured by the SPI spectrometer onboard the INTEGRAL satellite [37. Since Fig. 6 was done with a cross section of $\langle\sigma v\rangle=3 \cdot 10^{-26} \mathrm{~cm}^{3} \mathrm{~s}^{-1}$, such a limit translates into a plateau below $m_{\chi} \sim 500 \mathrm{MeV}$, translating into a saturation of $\delta i_{\chi} \lesssim 10^{-8}$, much below the current and future sensitivities.

Invoking very cuspy profile with $\gamma>1$ appears be the only possibility left for DM to generate an observable SZ distortion of the CMB spectrum, but such an extreme configu- 


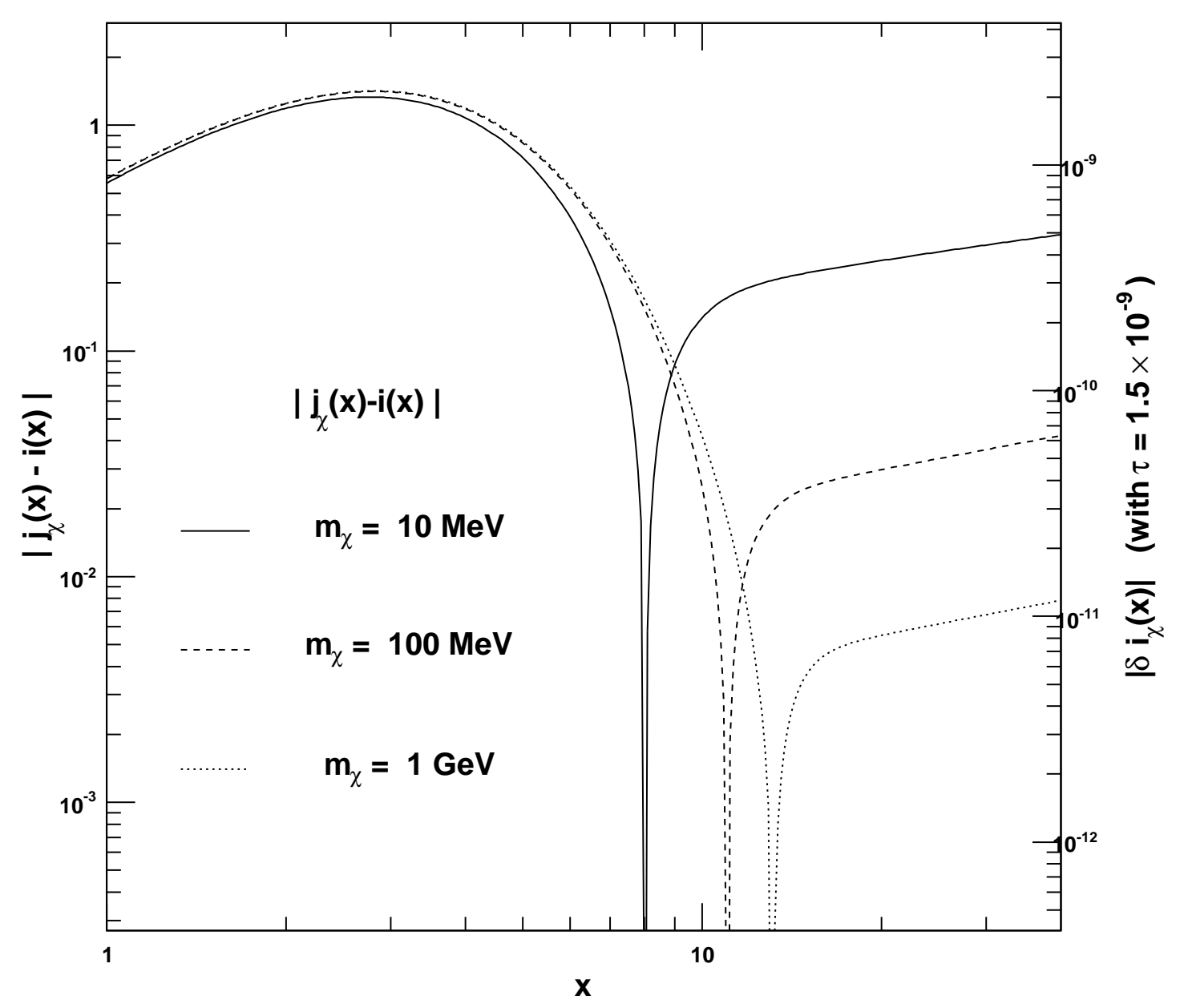

Figure 5: $\left|\delta i_{\chi}(x)\right| / \tau_{\chi}=\left|j_{\chi}(x)-i(x)\right|$ as a function of the reduced frequency $x$ for different WIMP masses, assuming direct annihilation in $e^{+} e^{-}$(identical for decaying DM, but corresponding to twice the masses). The right vertical axis provides the conversion to $\left|\delta i_{\chi}(x)\right|$ for an optical depth of $\tau=1.5 \times 10^{-9}$, corresponding to a configuration with $1 \mathrm{GeV}$ annihilating DM with an NFW profile inside our template cluster observed with an angular resolution of $10^{\prime \prime}$ - see Eq. (3.11) and Fig. 田.

ration (i) is hardly motivated from theoretical and observational constraints and (ii) will even be shown, in the next section, to have limited effect due to spatial diffusion. Last but not least, one should not forget about the additional foreground coming from other relativistic electrons also injected in clusters from standard astrophysical sources [32]. In any case, any attempt of DM interpretation of any SZ imprints would seem daring, at least in our view. 


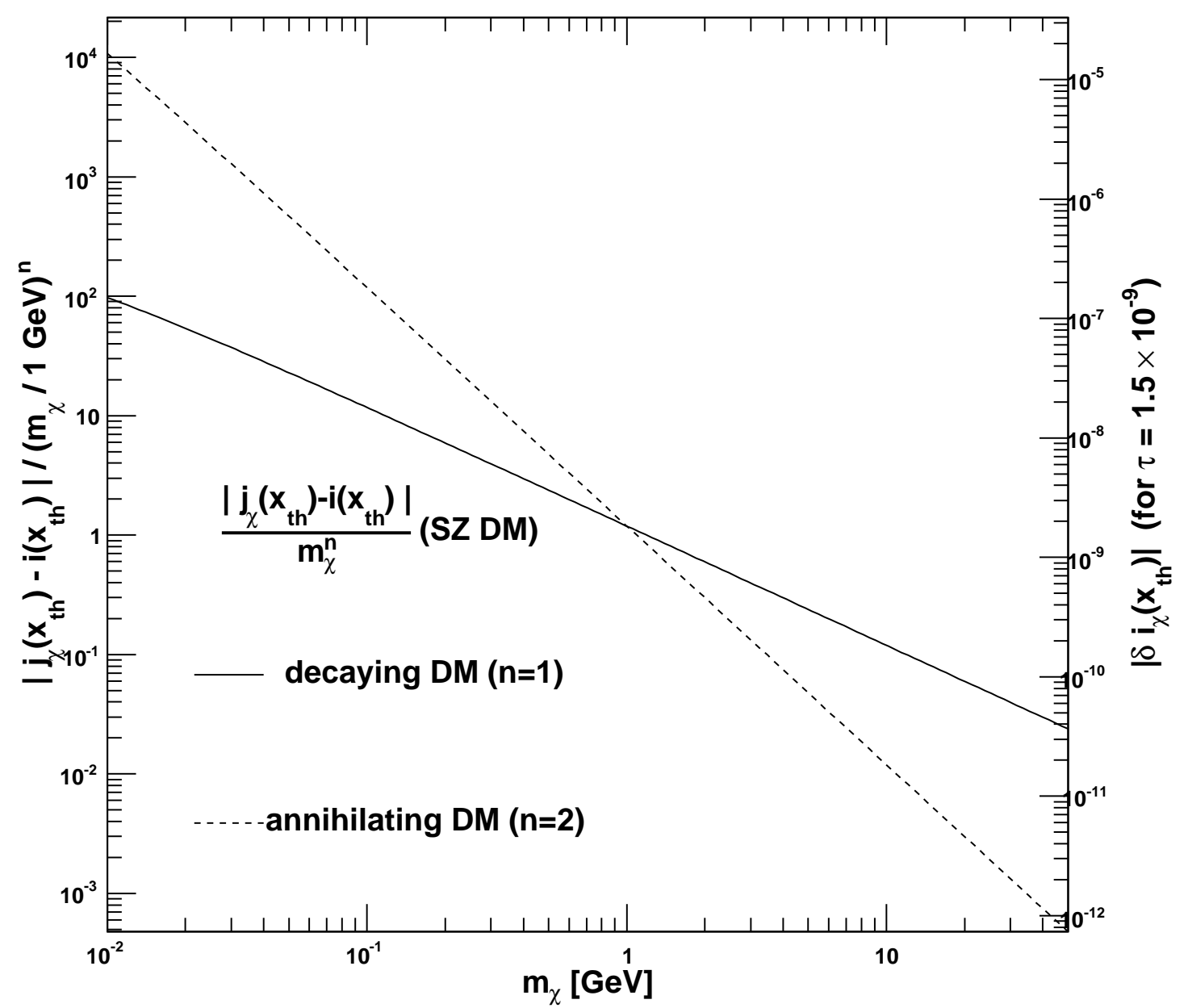

Figure 6: $\delta i_{\chi}\left(x_{\mathrm{th}}\right) /\left(m_{\chi}^{n} \tau_{\chi}\right)$ as a function of the DM particle mass $m_{\chi}$, for $x_{\mathrm{th}}=4$ (left vertical axis). The right vertical axis provides the conversion to $\left|\delta i_{\chi}\left(x_{\mathrm{th}}\right)\right|$ for an optical depth of $\tau=1.5 \times 10^{-9}$, corresponding to a configuration with annihilating DM with an NFW profile inside our template cluster observed with an angular resolution of $10^{\prime \prime}-$ see Eq. (3.11) and Fig. 目.

\section{Impact of spatial diffusion: analytical insights}

So far, we have assumed that the equilibrium electron density was set only from energy losses and neglected the spatial transport, which was presented as a reasonable hypothesis in [7] for angular resolutions $\lesssim 1^{\prime}$. Nevertheless, as discussed above, angular resolutions down to $1^{\prime \prime}$, as expected in future experiments, correspond to quite small spatial scales for typical nearby clusters, so that the relevance of such an assumption can be questioned.

If we include spatial diffusion, then Eq. (3.3) must be rewritten in terms of a current 
conservation equation [38]

$$
-\vec{\nabla}\left(K_{r} \vec{\nabla} \frac{d n}{d E}\right)-\partial_{E}\left(b(E) \frac{d n}{d E}\right)=\mathcal{Q}(\vec{x}, E) .
$$

$K_{r}$ is the diffusion coefficient that characterizes the stochastic transport of electrons caused by their diffusion on the magnetic inhomogeneities of the intracluster medium. We will assume this coefficient to depend on the energy only $K_{r} \equiv K_{r}(E)$. This equation can be solved by looking for the associated Green function, which is readily found in infinite 3D space:

$$
\mathcal{G}\left(\vec{x}, E \leftarrow \vec{x}_{s}, E_{s}\right)=\frac{1}{b(E) \pi^{\frac{3}{2}} \lambda^{3}} \exp \left\{-\frac{\left(\vec{x}_{s}-\vec{x}\right)^{2}}{\lambda^{2}}\right\},
$$

where $\lambda$ is a characteristic propagation scale defined as

$$
\lambda^{2}\left(E, E_{s}\right) \equiv 4 \int_{E}^{E_{s}} d E^{\prime} \frac{K\left(E^{\prime}\right)}{b\left(E^{\prime}\right)} .
$$

The electron density at any position $\vec{x}$ and energy $E$ in the cluster is then given by

$$
\frac{d n}{d E}(\vec{x}, E)=\int_{E}^{n m_{\chi} / 2} d E_{s} \int d^{3} \vec{x}_{s} \mathcal{G}\left(\vec{x}, E \leftarrow \vec{x}_{s}, E_{s}\right) \mathcal{Q}\left(\vec{x}_{s}, E_{s}\right)
$$

We can remark that we recover the diffusion-less case in the limit of vanishingly small propagation scale $\lambda \rightarrow 0$, since in this case we have $\mathcal{G}\left(E, \vec{x} \leftarrow \vec{x}_{s}\right) \leftrightarrow \delta^{3}\left(\vec{x}-\vec{x}_{s}\right) / b(E)$. For a monochromatic injection of electrons from DM annihilation or decay, the diffusion-less limit is therefore recovered when $E \rightarrow n m_{\chi} / 2$. More generally however, since the Green function exhibits a Gaussian behavior of typical scale $\lambda$, the electron density is expected to be smeared out over this scale, which we actually demonstrate below.

The Green function appearing in Eq. (5.2) was derived assuming diffusion in an infinite $3 \mathrm{D}$ space, which is obviously not the case since clusters have finite sizes. This still holds provided the propagation scale $\lambda$ is much lower than the typical size of the cluster, and while electron injection at the border of the object is irrelevant compared to injection in the more central regions. If set by magnetic inhomogeneities, the diffusion coefficient can be expressed as a function of the regular component of the magnetic field. Although it is absolutely not clear whether or not a Kolmogorov spectrum is relevant to describe magnetic turbulence in clusters, since it is not the case at the Galactic scale, it is still conventional to adopt such a behavior for the diffusion coefficient [39]:

$$
K_{r}(E)=K_{0}\left[\frac{l_{B}}{20 \mathrm{kpc}}\right]^{2 / 3}\left[\frac{B}{\mu \mathrm{G}}\right]^{-1 / 3}\left[\frac{E}{\mathrm{GeV}}\right]^{1 / 3},
$$

where $B$ is the magnetic field and $l_{B}$ its coherence length. With the above values, which we will use in the following, the normalization of the diffusion coefficient can set to $K_{0} \sim$ $2.3 \times 10^{29} \mathrm{~cm}^{2} \mathrm{~s}^{-1}$ 39]. Since the typical energy loss timescale is of order $\tau_{\text {loss }} \sim 10^{16} \mathrm{~s}$, we have $\lambda \sim 2 \sqrt{K_{0} \tau_{\text {loss }}} \sim 30 \mathrm{kpc} \ll r_{s}$, which is actually quite large though still smaller 
than the typical scale radii of clusters - note that this diffusion scale will slightly decrease with energy above $1 \mathrm{GeV}$, when the inverse Compton dominates over the Coulomb losses. Therefore, because the injection rate of electrons is expected to dominate within a volume set by $r_{s} \ll R_{\text {vir }}$ and since $\lambda \ll r_{s}$, we can safely disregard the radial boundary condition and use the infinite $3 \mathrm{D}$ solution written above.

The source term $\mathcal{Q}$ is defined in Eq. (3.1). To simplify the discussion, we will further suppose that DM annihilates or decays into electron-positron pairs, so that $N_{0}=2$ and $F\left(E_{s}\right)=\delta\left(E_{s}-n m_{\chi} / 2\right)-n=2$ in the case of annihilation, 1 in the case of decay. In this fiducial instance, the propagation scale only depends on the energy $E, \lambda=\lambda\left(E, n m_{\chi} / 2\right)-$ see Eq. (5.3). Therefore, the electron density at any cluster radius $r$ and energy $E \leq n m_{\chi} / 2$ reduces to

$$
\frac{d n}{d E}(r, E)=\frac{4 \alpha_{n} \rho_{s}^{n}}{b(E) \sqrt{\pi} \lambda^{3}} \int_{-1}^{1} d \mu^{\prime} \int d r^{\prime} r^{\prime 2} \exp \left\{-\frac{r^{\prime 2}+r^{2}-2 r r^{\prime} \mu^{\prime}}{\lambda^{2}}\right\} f_{\gamma}^{n}\left(r^{\prime}\right)
$$

where we have taken advantage of the spherical symmetry of the source term, the radial dependence $f_{\gamma}\left(r^{\prime}\right)$ of which has been defined in Eq. (3.2). The integral over the cosine $\mu^{\prime}$ is straightforward, and we are left with:

$$
\begin{aligned}
\frac{d n}{d E}(r, E) & =\frac{2 \alpha_{n} \rho_{s}^{n}}{b(E) \sqrt{\pi} \lambda r} \sum_{k=0}^{k=1}(-1)^{k} \int d r^{\prime} r^{\prime} f_{\gamma}^{n}\left(r^{\prime}\right) \exp \left\{-\frac{\left(r-(-1)^{k} r^{\prime}\right)^{2}}{\lambda^{2}}\right\} \\
& \simeq \frac{2 \alpha_{n} \rho_{s}^{n} r_{s}^{n \gamma}}{b(E) \sqrt{\pi} \lambda r} \sum_{k=0}^{k=1}(-1)^{k} \int_{r-\lambda}^{r+\lambda} d r^{\prime}\left|r^{\prime}\right|^{1-n \gamma} \exp \left\{-\frac{\left(r-(-1)^{k} r^{\prime}\right)^{2}}{\lambda^{2}}\right\} .
\end{aligned}
$$

In the last line, we have employed the approximation given in Eq. (3.15), where the DM profile is taken as a simple power law; indeed, we are mostly interested, again, in the SZ contribution from the densest central parts of the cluster. Moreover, we have restricted the integral range to $[r-\lambda ; r+\lambda]$, accounting for the fact that electrons coming from distances above $\lambda$ are Gaussianly depleted, consistently with Eq. (5.2); we remind that $\lambda \sim 10 \mathrm{kpc}$. In the very central region of the cluster and within this range for $r^{\prime}$, the arguments in the exponentials $\left(r-(-1)^{k} r^{\prime}\right) \lesssim \lambda$, so that we can expand the integral to get more insights on the physics at stake. To the first order, the result is analytical, and we find after integration:

$$
\frac{d n}{d E}(r, E) \simeq \frac{8 \alpha_{n} \rho_{s}^{n} r_{s}^{n \gamma}}{b(E) \sqrt{\pi} \lambda^{3}} \frac{(r+\lambda)^{3-n \gamma}}{(3-n \gamma)}\left[1-\operatorname{sign}(r-\lambda) \frac{|r-\lambda|^{3-n \gamma}}{(r+\lambda)^{3-n \gamma}}\right],
$$

where we let the reader derive the logarithmic expression that arises when $3-n \gamma=0$. This result is very interesting, since it states that at the center of the cluster, i.e. $r=0$, the electron density is no longer diverging like $r^{-n \gamma}$ when diffusion occurs, but instead behaves like:

$$
\frac{d n}{d E}(0, E) \approx \frac{16 \alpha_{n} \rho_{s}^{n}(3-n \gamma)^{-1}}{b(E) \sqrt{\pi}}\left[\frac{r_{s}}{\lambda}\right]^{n \gamma}
$$

As expected, the electron density scales like $\lambda^{-n \gamma}$, where $\lambda$ is the diffusion scale, but we have grounded this intuition on a rather rigorous demonstration. Of course, this approximation 
is not valid for vanishingly small values of $\lambda$. This means that at any energy but the maximal energy injected from DM annihilation or decay - when $\lambda$ is sizable - the actual electron density is not governed by the (squared) DM density itself, but is diluted away over a region delineated by $\lambda$.

This has important consequences for the SZ calculation. Indeed, we have computed the optical depth in the diffusion-less approximation, showing that it was significantly increasing with angular resolution for cuspy profiles. Nevertheless, we have just demonstrated that the electron density should in fact saturate within a scale fixed by $\sim \lambda$, leading to the saturation of the optical depth itself, independently of the angular resolution. For $\lambda \sim 10$ $\mathrm{kpc}$, this would correspond to an angular size of $\sim 40^{\prime \prime}$ for our template cluster, as depicted in Fig. 1, which means that instruments with better resolution than this value would not improve the detection potential.

The full SZ computation is slightly different when spatial diffusion is considered, since we can no longer factorize the energy and the spatial parts as we did in Eqs. (3.5, 3.5, 3.6), but must use Eq. (5.4) to compute the the optical depth. Nevertheless, up to a good approximation valid in the monochromatic injection case, we can associate an energydependent core radius of size $\lambda\left(E, n m_{\chi} / 2\right)$ to each halo model. Given the values that we used for the diffusion coefficient and for the energy loss rate, this would lead to a plateau in the optical depth curves for scales below $\sim 10 \mathrm{kpc}$, as also found in the final published version of [10]. Since our spectral analysis was performed for an NFW profile and an angular resolution of $10^{\prime \prime}$, the full spatial diffusion calculation would not only decrease the predicted amplitude by a factor of $\sim 2$, as seen from Fig. 1, but also make this result constant for any better angular resolution. Should have we done this exercise with a Moore profile, we would have overshot the actual amplitude by one order of magnitude.

\section{Conclusion}

In this paper, we have revisited the prospect for radio experiments, like Planck or ALMA, for observing SZ distortions of the CMB spectrum due to the presence of relativistic electrons induced by DM annihilation or decay. Whenever possible, we have provided analytical grounds to the discussion.

In Sec. 3, we have first focused on the most important physical weight on the distortion amplitude, the optical depth associated with the electron density (the thermal background as well as electrons induced by DM annihilation or decay). We have considered typical values for the DM particle properties and a generic template cluster located at a distance of $50 \mathrm{Mpc}$ (Coma lies at $\sim 90 \mathrm{Mpc}$ ), with features very close to those observed in $\mathrm{N}$ body simulations and a thermal electron population with a density of $n_{e, \text { th }}=0.01 \mathrm{~cm}^{-3}$, consistent with most of X-ray observations.

In the frame of the diffusion-less limit proposed in [7], we have stressed the importance of the angular resolution inside which the SZ signal has to be averaged, showing that even for very optimistic values of $\sim 1^{\prime \prime}$, the thermal electron optical depth almost always strongly dominate over that of the DM-induced electrons. This actually means that the thermal SZ completely overcomes the DM SZ at all frequencies but the thermal transition frequency. 
In addressing the angular resolution issue, we have derived simple and useful analytical approximations of the line-of-sight integral (Eqs. 3.16 and 3.20, the latter being more accurate) that can be used to compute the optical depth for any decaying or annihilating DM model. Actually, they are also useful for quick predictions of $\gamma$-ray fluxes from DM annihilation or decay in any extragalactic objects, subhalos, dwarf spheroidals, galaxies or clusters.

Then, we have performed the full spectral analysis of the SZ distortion due to DMinduced electrons in Sec. 1 , showing that it can hardly exceed the $\mu \mathrm{K}$ level in terms of temperature fluctuations, even for e.g. very light DM particles, which are further constrained by $511 \mathrm{keV}$ observations of the Galactic center. Unconventional very cuspy cluster halo profiles and very large annihilation cross sections or decay rates that would escape other astrophysical contraints for some reasons might still lead to signal close to future sensitivities. However, such configurations are not supported by current studies in each of the specific inputs and are therefore quite unlikely.

Finally, we have discussed the impact of considering the spatial transport of electrons in clusters in Sec. 5, providing again analytical results. We have demonstrated that the diffusion-less limit is actually not valid in the cluster center for SZ computations with small angular resolutions because the electron density is actually smoothed over a scale set by the transport scale. This scale is small only for energies close to their injection values,

and are therefore sizable over most the whole energy range but for $E \rightarrow m_{\chi}$ in the case of annihilating DM. Given the propagation parameters used in our analysis, this scale is of order $\sim 10 \mathrm{kpc}$, below which the optical depth saturates. This means that improving the angular resolution below that scale will not improve the detection potential, unfortunately. Our results are in good agreement with those obtained in [10], to which they provide deeper analytical grounds. Reversely, we cannot support the statements made in [5, 6, [7] about the promising potential of SZ observations for indirect detection of DM.

\section{Acknowledgments}

We are indebted to M. Langer and M. Fairbairn, who initiated the early stages of this work with us. JL is grateful to LAPTH for hospitality during different stages of this study, and acknowledges financial support by the French ANR project ToolsDMColl (BLAN072-194882).

\section{References}

[1] G. Jungman, M. Kamionkowski, and K. Griest, Supersymmetric dark matter, Phys. Rept. 267 (Mar., 1996) 195-373, arXiv:hep-ph/9506380.

[2] H. Murayama, Physics Beyond the Standard Model and Dark Matter, ArXiv e-prints (Apr., 2007) arXiv:0704.2276.

[3] C. Bœhm and P. Fayet, Scalar dark matter candidates, Nuclear Physics B 683 (Apr., 2004) 219-263, arXiv:hep-ph/0305261. 
[4] P. Salati, Indirect and direct dark matter detection, Proceedings of Science Cargese 2007 (2007) 009.

[5] S. Colafrancesco and B. Mele, Neutralinos and the Origin of Radio Halos in Clusters of Galaxies, Astrophys. J. 562 (Nov., 2001) 24-41, [arXiv:astro-ph/0008127].

[6] S. Colafrancesco, SZ effect from Dark Matter annihilation, Astron. $\& 3$ Astrophys. 422 (July, 2004) L23-L27, arXiv:astro-ph/0405456].

[7] S. Colafrancesco, S. Profumo, and P. Ullio, Multi-frequency analysis of neutralino dark matter annihilations in the Coma cluster, Astron. \& Astrophys. 455 (Aug., 2006) 21-43, arXiv: astro-ph/0507575.

[8] R. A. Sunyaev and Y. B. Zeldovich, The Observations of Relic Radiation as a Test of the Nature of X-Ray Radiation from the Clusters of Galaxies, Comments on Astrophysics and Space Physics 4 (Nov., 1972) 173-+.

[9] R. A. Sunyaev and I. B. Zeldovich, Microwave background radiation as a probe of the contemporary structure and history of the universe, Ann. Rev. Astron. $\& 5$ Astrophys. 18 (1980) 537-560.

[10] Q. Yuan, X. Bi, F. Huang, and X. Chen, Dark matter annihilation and non-thermal Sunyaev-Zel'dovich effect: I. Galaxy cluster, Journal of Cosmology and Astro-Particle Physics 10 (Oct., 2009) 13-+, arXiv:0902.4294.

[11] The Planck Collaboration, The Scientific Programme of Planck, ArXiv Astrophysics e-prints (Apr., 2006) arXiv:astro-ph/0604069.

[12] Science with ALMA, Science with the Atacama Large Millimeter Array: A New Era for Astrophysics, Astrophysics and Space Science 313 (Jan., 2008) 1-335.

[13] A. Wootten and A. R. Thompson, The Atacama Large Millimeter/submillimeter Array, ArXiv e-prints (Apr., 2009) arXiv:0904.3739.

[14] M. Birkinshaw, The Sunyaev-Zel'dovich effect, Phys. Rept. 310 (Mar., 1999) 97-195, arXiv: astro-ph/9808050.

[15] E. L. Wright, Distortion of the microwave background by a hot intergalactic medium, Astrophys. J. 232 (Sept., 1979) 348-351.

[16] S. Chandrasekhar, Radiative transfer. Oxford, Clarendon Press, 1950., 1950.

[17] A. Challinor and A. Lasenby, Relativistic Corrections to the Sunyaev-Zeldovich Effect, Astrophys. J. 499 (May, 1998) 1-+, [arXiv:astro-ph/9711161].

[18] N. Itoh, Y. Kohyama, and S. Nozawa, Relativistic Corrections to the Sunyaev-Zeldovich Effect for Clusters of Galaxies, Astrophys. J. 502 (July, 1998) 7-+, arXiv:astro-ph/9712289.

[19] S. Y. Sazonov and R. A. Sunyaev, Cosmic Microwave Background Radiation in the Direction of a Moving Cluster of Galaxies with Hot Gas: Relativistic Corrections, Astrophys. J. 508 (Nov., 1998) 1-5.

[20] A. Stebbins, Extensions to the Kompaneets Equation and Sunyaev-Zel'dovich Distortion, ArXiv Astrophysics e-prints (Sept., 1997) arXiv:astro-ph/9709065.

[21] A. D. Dolgov, S. H. Hansen, S. Pastor, and D. V. Semikoz, Spectral Distortion of Cosmic Microwave Background Radiation by Scattering on Hot Electrons: Exact Calculations, Astrophys. J. 554 (June, 2001) 74-84, arXiv:astro-ph/0010412. 
[22] C. Bœhm and J. Lavalle, Clarifying the covariant formalism for the Sunyaev-Zel'dovich effect due to relativistic nonthermal electrons, Phys. Rev. D 79 (Apr., 2009) 083505-+, arXiv:0812.3282.

[23] S. Nozawa and Y. Kohyama, Analytical study on the Sunyaev-Zeldovich effect for clusters of galaxies, Phys. Rev. D 79 (Apr., 2009) 083005-+, arXiv:0902.2595.

[24] L. Bergström, P. Ullio, and J. H. Buckley, Observability of gamma rays from dark matter neutralino annihilations in the Milky Way halo, Astroparticle Physics 9 (Aug., 1998) 137-162, arXiv:astro-ph/9712318.

[25] H. Zhao, Analytical models for galactic nuclei, MNRAS 278 (Jan., 1996) 488-496, arXiv: astro-ph/9509122.

[26] J. F. Navarro, C. S. Frenk, and S. D. M. White, A Universal Density Profile from Hierarchical Clustering, Astrophys. J. 490 (Dec., 1997) 493, arXiv:astro-ph/9611107.

[27] B. Moore, F. Governato, T. Quinn, J. Stadel, and G. Lake, Resolving the Structure of Cold Dark Matter Halos, Astrophys. J. Lett. 499 (May, 1998) L5, arXiv:astro-ph/9709051.

[28] J. S. Bullock, T. S. Kolatt, Y. Sigad, R. S. Somerville, A. V. Kravtsov, A. A. Klypin, J. R. Primack, and A. Dekel, Profiles of dark haloes: evolution, scatter and environment, MNRAS 321 (Mar., 2001) 559-575, arXiv:astro-ph/9908159.

[29] V. S. Berezinsky, A. V. Gurevich, and K. P. Zybin, Distribution of dark matter in the galaxy and the lower limits for the masses of supersymmetric particles, Physics Letters B 294 (Nov., 1992) 221-228.

[30] G. Gentile, P. Salucci, U. Klein, D. Vergani, and P. Kalberla, The cored distribution of dark matter in spiral galaxies, MNRAS 351 (July, 2004) 903-922, arXiv:astro-ph/0403154.

[31] J. F. Navarro, A. Ludlow, V. Springel, J. Wang, M. Vogelsberger, S. D. M. White, A. Jenkins, C. S. Frenk, and A. Helmi, The Diversity and Similarity of Simulated Cold Dark Matter Halos, ArXiv e-prints (Oct., 2008) arXiv:0810.1522].

[32] T. A. Enßlin and C. R. Kaiser, Comptonization of the cosmic microwave background by relativistic plasma, Astron. \& Astrophys. 360 (Aug., 2000) 417-430, arXiv: astro-ph/0001429.

[33] C. L. Sarazin, The Energy Spectrum of Primary Cosmic-Ray Electrons in Clusters of Galaxies and Inverse Compton Emission, Astrophys. J. 520 (Aug., 1999) 529-547, arXiv: astro-ph/9901061.

[34] V. L. Ginzburg, Theoretical physics and astrophysics. Oxford: Pergamon, 1979.

[35] C. Boehm, D. Hooper, J. Silk, M. Casse, and J. Paul, MeV Dark Matter: Has It Been Detected?, Physical Review Letters 92 (Mar., 2004) 101301-+, arXiv:astro-ph/0309686.

[36] Y. Ascasibar, P. Jean, C. Boehm, and J. Knödlseder, Constraints on dark matter and the shape of the Milky Way dark halo from the 511-keV line, MNRAS 368 (June, 2006) 1695-1705, arXiv: astro-ph/0507142].

[37] J. Knödlseder, P. Jean, V. Lonjou, G. Weidenspointner, N. Guessoum, W. Gillard, G. Skinner, P. von Ballmoos, G. Vedrenne, J. Roques, S. Schanne, B. Teegarden, V. Schönfelder, and C. Winkler, The all-sky distribution of $511 \mathrm{keV}$ electron-positron annihilation emission, Astron. \& Astrophys. 441 (Oct., 2005) 513-532, arXiv: astro-ph/0506026. 
[38] V. L. Ginzburg and S. I. Syrovatskii, The Origin of Cosmic Rays. The Origin of Cosmic Rays, New York: Macmillan, 1964, 1964.

[39] P. Blasi and S. Colafrancesco, Cosmic rays, radio halos and nonthermal X-ray emission in clusters of galaxies, Astroparticle Physics 12 (Nov., 1999) 169-183,

arXiv: astro-ph/9905122. 